\title{
Connectivity Times in Vehicular Networks
}

\author{
Dieter Fiems and Alexey Vinel
}

\begin{abstract}
We propose an analytical model that accurately captures the service advertisement and access mechanisms in IEEE 1609.4/IEEE 802.11p multi-channel vehicular networks where road-side units announce non-safety services to passing vehicles on a dedicated channel. For a drive-thru scenario, we calculate various performance measures including the service discovery probability, the mean time till discovery and the channel utilization.
\end{abstract}

\section{INTRODUCTION}

By leveraging remote connectivity supplied by Road-Side Units (RSUs), vehicles can exchange environmental/traffic data gathered by on-board and on-the-road sensors, retrieve/update maps, or access traditional Internet services like web browsing and cloud access via vehicle-to-roadside (V2R) communications. In a drive-thru scenario [1], where moving vehicles spend at most a couple of minutes in the coverage area of a RSU, V2R communication links are expected to be shortlived and intermittent, due to the mobility of the vehicles and the high cost to deploy a ubiquitous roadside infrastructure.

Such scenarios pose specific performance issues which have attracted attention in the research community. Spatially coordinated channel access in a drive-thru scenario is presented in [2]. Assuming that the RSU coverage area can be divided in zones by achievable throughput, the authors optimize the assignment of zones to vehicles such that the overall system throughput is maximized. In addition, [3] investigates a stochastic model to assess the mean packet service time, the queue length and the throughput per vehicle in a drive-thru scenario.

The modeling frameworks above make abstraction of the implementation of the service discovery process, which is crucial to make the best of V2R connectivity opportunities. In particular, the time between service announcements is the key to quick service discovery. If this time is small, the service discovery time will be small, while the probability to detect the service will be high. There is however a trade-off. As the RSU has to switch its transceiver from the channel where services are provided to the advertisement channel, connected vehicles experience disruption during advertisements, yielding a considerably reduction of the channel utilization.

The service discovery protocol is defined in the IEEE 1609.4 vehicular communications standard, but an adequate analytical model for its performance evaluation is missing in the literature. While the mean service discovery time and the service

D. Fiems is with Ghent University, Dep. TELIN, St-Pietersnieuwstraat 41, 9000 Gent, Belgium

A. Vinel is with Halmstad University, School of ITE, Kristian IV:s väg 3, SE-30118 Halmstad, Sweden

This work has been partly supported by EU COST Action CA15127 RECODIS "Resilient communication services protecting end-user applications from disaster-based failures", Knowledge Foundation (KKS) in Sweden and ELLIIT Strategic Research Network. channel utilization are derived in [4], this work assumes that the error rate is constant and does not depend on the position of the vehicle in the RSU coverage area. This is however contrary to the nature of a drive-thru scenario where vehicles experience changing error rates while traversing the coverage area of the RSU [2]; also see [5] for supporting field measurements. In this letter, we introduce an analytical model for studying the discovery process which accounts for the changing error rates. Our numerical results reveal that this additional modelling detail is needed for an accurate performance evaluation.

\section{ANALYTICAL FRAMEWORK}

We consider a vehicle moving at a constant speed $v$ along a path, experiencing location dependent bit error probabilities (or rates - BER). Without loss of generality, we assume that at time $t=0$ the vehicle starts in position $z=0$, and that the vehicle is outside the range of the RSU at position $z=Z$.

The RSU regularly announces the available drive-thru services, by transmitting a broadcast Service Announcement Message (SAM) indicating services and the channel on which they can be accessed. Once an announcement is received, an interested vehicle tunes its transceiver to the announced channel and accesses the offered service(s) while it remains in the coverage area of the RSU. The IEEE 1609.4 RSU periodically switches the transceiver between the advertising channel (e.g. Service Channel $1-\mathrm{SCH} 1$ ) and the advertised channel (e.g. SCH 2-4) with period $\tau$, referred to as the SAM period, as depicted in Figure 1.

During each SAM period, the RSU cannot provide service to the vehicles while tuned into the advertising channel. This service-disruption time has a random duration $\mathrm{X}$. The service period then corresponds to the remainder of the SAM period and is denoted by $\mathbf{Y}=\tau-\mathbf{X}$. Note that $\mathbf{X}$ is random as the IEEE 802.11p backoff delay is random. Apart from the backoff delay $\mathbf{B}$, the service disruption period includes the times $T_{\mathrm{sw}}$ needed to switch to and from the advertising channel and the time to transmit the SAM message $t_{0}$, that is,

$$
\mathbf{X}=\mathbf{B}+2 T_{\mathrm{sw}}+t_{0} .
$$

The time to transmit the SAM message on the advertising channel includes mandatory idle time. We have, $t_{0}=T_{h}+$ $L / R+\mathrm{SIFS}+\mathrm{AIFSN} \cdot \sigma$, where $T_{h}$ denotes the SAM header duration, $L$ denotes the SAM payload size, $R$ denotes the SAM data rate, SIFS denotes the Short Inter-Frame Space, $\sigma$ denotes aSlotTime and AIFSN denotes the Arbitration InterFrame Space Number specified in IEEE 802.11p.

Correct reception of the SAM message depends on the BER, which is assumed to depend on both location and speed of the vehicle. Let $b(z, v)$ be the BER when the vehicle is in position $z$ with speed $v$. For ease of presentation, we further assume 
that $b(z, v)=1$ for $z>Z$. For further reference, let $\mathbf{I}$ be the indicator that the SAM message is received with errors.

Given the assumptions above, we focus on computing the discovery probability and the mean discovery delay, conditional on service discovery. The discovery probability is the probability that the service is discovered while the vehicle traverses the coverage area, while the discovery delay is the time between the moment the vehicle enters the coverage area of the RSU, and the moment the SAM message is successfully received. We also calculate the utilization $\rho$. This is the fraction of the time which is available for accessing services.

\section{A. Service disruption time and utilization}

We first focus on the mean backoff delay $E[\mathbf{B}]$. As the backoff delay does not depend on the error model, neither location nor velocity influences the backoff delay. Approximating the backoff procedure with a contention window of $W$ slots by a sequence of independent transmissions with probabilities $2 /(W+1)$, we find the collision probability,

$$
p_{0}=1-\left(1-2(W+1)^{-1}\right)^{N},
$$

where $N$ denotes the number of contending nodes. With a slight abuse of notation, let $\mathbf{B}_{w}$ be the backoff delay for window size $w$, and for collision probability $p_{0}$ as given above. We find,

$$
\mathrm{E}\left[\mathbf{B}_{w}\right]=\left(1-w^{-1}\right)\left(\bar{p}_{0} \sigma+p_{0} t_{0}+\mathrm{E}\left[\mathbf{B}_{w-1}\right]\right),
$$

with $\bar{p}_{0}=1-p_{0}$. This recursion follows from the observation that the backoff delay is zero with probability $1 / w$ while with probability $(1-1 / w)$ it is equal to the sum of the backoff delay with window size $w-1$ and either the time slot $\sigma_{0}$ (if no other user sends) or the SAM transmission time $t_{0}$ (if another user sends). As transmission starts when the window size is 1 , we have $E\left[\mathbf{B}_{1}\right]=0$. We can then explicitly solve the recursion, yielding,

$$
\mathrm{E}[\mathbf{B}] \doteq \mathrm{E}\left[\mathbf{B}_{W}\right]=\frac{1}{2}(W-1)\left(\bar{p}_{0} \sigma+p_{0} t_{0}\right) .
$$

By equation (1), we further find the following expression for the mean service disruption time,

$$
\mathrm{E}[\mathbf{X}]=\frac{1}{2}(W-1)\left(\bar{p}_{0} \sigma+p_{0} t_{0}\right)+2 T_{\mathrm{sw}}+t_{0} .
$$

Moreover, given $E[\mathbf{X}]$, we can also calculate the fraction of time the service is available, say the utilization $\rho$. As the service is periodically disrupted with period $\tau$, we have

$$
\rho=(\tau-\mathbf{E}[\mathbf{X}]) \tau^{-1} .
$$

\section{B. SAM failure probability}

We now focus on the SAM failure probability. Recall that I is the indicator that the SAM message is received with errors. We therefore have $p(z, v)=\mathrm{E}_{z, v}[\mathbf{I}], \mathrm{E}_{z, v}$ being the expectation operator given that the vehicle is in position $z$ with velocity $v$ at the start of the SAM period.

The SAM message is correctly received when there is no collision, and when all bits of the payload are correctly received. We hereby neglect the probability that the header of the

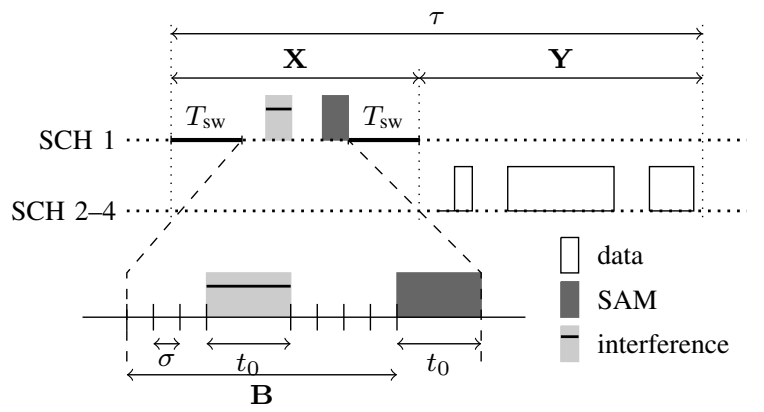

Fig. 1. Service Announcement Message (SAM) period.

SAM message is not received correctly, as this header benefits from additional protection. The SAM failure probability for a vehicle with velocity $v$ at position $z$ when the transmission of the SAM message starts is,

$$
p_{t}(z, v)=1-\bar{p}_{0} \prod_{k=0}^{L-1}\left(1-b\left(z+T_{h} v+k v / R, v\right)\right) .
$$

The SAM failure probability depends on the backoff delay. Indeed, the location changes during the backoff delay. Therefore, let $p_{b}(z, v, w)$ be the failure probability when the vehicle is in position $z$ with speed $v$ at the start of the backoff period with window size $w$. Analogous to the the mean backoff delay calculations, we find a recursion for $p_{b}(z, v, w)$,

$$
\begin{aligned}
& p_{b}(z, v, w)=w^{-1} p_{t}(z, v)+\left(1-w^{-1}\right) \times \\
& \quad\left(\bar{p}_{0} p_{b}(z+v \sigma, v, w-1)+p_{0} p_{b}\left(z+v t_{0}, v, w-1\right)\right) .
\end{aligned}
$$

As the backoff delay is zero for $w=1$, we have, $p_{b}(z, v, 1)=$ $p_{t}(z, v)$. As such, the recursion above allows for numerically determining $p_{b}(z, v, w)$ for any $z, v$ and $w$.

Accounting for the switching time $T_{\mathrm{sw}}$, and suppressing the dependency on $W$ in the notation, we finally find the failure probability of the SAM message when the car is in position $z$ with velocity $v$ at the start of the SAM period,

$$
p(z, v)=\mathrm{E}_{z, v}[\mathbf{I}]=p_{b}\left(z+T_{\mathrm{sw}} v, v, W\right) .
$$

\section{Conditional service disruption time}

We now consider the expectation $\mathrm{E}_{z, v}[\mathbf{X I}]$ which relates to the mean service disruption time, conditioned on the transmission failure of the SAM message as follows,

$$
\mathrm{E}_{z, v}[\mathbf{X} \mid \mathrm{SAM} \text { not received }]=\frac{\mathrm{E}_{z, v}[\mathbf{X I}]}{p(z, v)} .
$$

Define $x(z, v) \doteq \mathrm{E}_{z, v}[\mathbf{X I}]$. In view of equation (1) we have,

$$
\begin{aligned}
x(z, v) & =\mathrm{E}_{z, v}[\mathbf{I}]\left(2 T_{\mathrm{sw}}+t_{0}\right)+\mathrm{E}_{z, v}[\mathbf{B I}] \\
& =p(z, v)\left(2 T_{\mathrm{sw}}+t_{0}\right)+\mathrm{E}_{z, v}[\mathbf{B I}] .
\end{aligned}
$$

Let $B(z, v, w)$ be the mean of $\mathbf{B I}$ given that the vehicle is position $z$ with speed $v$ at the start of the backoff period with window size $w$. Accounting for the switching time $T_{\mathrm{sw}}$ and the window size $W$, we have,

$$
\mathrm{E}_{z, v}[\mathbf{B I}]=B\left(z+v T_{\mathrm{sw}}, v, W\right) .
$$


It now remains to calculate $B(z, v, w)$. To this end, we proceed as for the mean backoff period and obtain a recursion by conditioning on the possible scenarios in the first time slot,

$$
\begin{aligned}
& B(z, v, w)=\left(1-w^{-1}\right) \times \\
& \left(\bar{p}_{0}\left(\sigma p_{b}(z+\sigma v, v, w-1)+B(z+\sigma v, v, w-1)\right)\right. \\
+ & \left.p_{0}\left(t_{0} p_{b}\left(z+t_{0} v, v, w-1\right)+B\left(z+t_{0} v, v, w-1\right)\right)\right) .
\end{aligned}
$$

Again, the recursion above allows for numerically determining $B(z, v, w)$ for any $z, v$ and $w$.

\section{Discovery probability}

We assume that the vehicle enters the coverage area at time 0 , and that the first (complete) SAM period starts at time $\mathbf{U}, \mathbf{U}$ being uniformly distributed between 0 and $\tau$. In other words, the vehicle enters the area at a random instant during the SAM period. Without loss of generality, we do not account for the partial SAM period on entry. We can make the probability of discovery during such a period arbitrarily small by extending the area, and assigning large BERs at large distances.

Let $p_{d}$ be the probability the service is discovered while the vehicle is in range. To simplify the calculations, we first calculate the probability $q_{k}(z, v)$ that the service is not discovered during the first $k$ (complete) SAM periods, assuming that the vehicle is in position $z$ when the first SAM period starts. Accounting for the SAM failure probabilities, the constant speed and the fixed length of the SAM periods, we have,

$$
q_{k}(z, v)=\prod_{\ell=0}^{k-1} p(z+\ell \tau v, v)
$$

where $p$ is defined in (3).

The vehicle leaves the range at location $Z$ (or at time $(Z-$ $z) / v)$ if it starts from position $z$ at time 0 . Hence, $\lfloor(Z-$ $z) / v / \tau\rfloor$ SAM periods start while the vehicle is in range. Now, the vehicle is in position $v \mathbf{U}$ when the first SAM period starts, such that the probability that the SAM message is received is,

$$
\begin{aligned}
p_{d} & =1-\int_{0}^{\tau} \frac{1}{\tau} q_{K(u)}(v u, v) d u \\
& \approx 1-\sum_{m=0}^{M-1} \frac{1}{M} q_{K(\tau m / M)}(v \tau m / M, v),
\end{aligned}
$$

with $K(u)=\lfloor(Z / v-u) / \tau\rfloor$. In the second expression, the uniform distribution of $\mathbf{U}$ is approximated by a discrete uniform distribution with $M$ points.

\section{E. Mean time till discovery}

The time till discovery now includes (i) the time $\mathbf{U}$ till the start of the first SAM period, (ii) the length of a SAM period during each SAM period where the SAM was not received and (iii) the service disruption time of the SAM period where the SAM message is received.

Let $\mathbf{D}$ denote the discovery time, where we define $\mathbf{D}$ to be equal to the residence time $Z / v$ of the vehicle in the coverage area of the RSU if service is not discovered. Let

\begin{tabular}{l|l}
\hline Parameter & Value \\
\hline Slot time $\sigma$ & $13 \mu \mathrm{s}$ \\
Contention window size $(W)$ & 15 \\
Interfering packets $(N)$ & $5-20$ \\
Short Inter-Frame Space (SIFS) & $32 \mu \mathrm{s}$ \\
Arbitration Inter-Frame Space Number (AIFSN) & 6 \\
SAM payload size $(L)$ & 300 bytes \\
SAM header duration $\left(T_{h}\right)$ & $40 \mu \mathrm{s}$ \\
SAM data rate $(R)$ & $6 \mathrm{Mbps}$ \\
SAM transmission time $\left(x_{0}\right)$ & $550 \mu \mathrm{s}$ \\
SAM period $(\tau)$ & $100 \mathrm{~ms}-1000 \mathrm{~ms}$ \\
Speed $(v)$ & $90 \mathrm{~km} / \mathrm{h}$ \\
Distance $(Z)$ & $1200 \mathrm{~m}$ \\
Channel switching delay $\left(T_{\mathrm{sw}}\right)$ & $4 \mathrm{~ms}$ \\
\hline
\end{tabular}

TABLE I

OVERVIEW OF THE DIFFERENT PARAMETERS

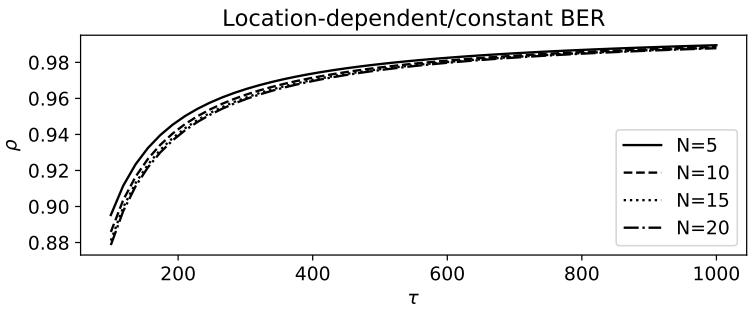

Fig. 2. Channel utilization $\rho$ vs. the SAM period $\tau$ for different numbers of vehicles $N$ as indicated.

$\mathbf{I}_{k}$ be the indicator function that the SAM message was not received during the $k$ th SAM period and let $\mathbf{X}_{k}$ be the service disruption time of the $k$ th SAM period. We have,

$$
\mathbf{D}=\mathbf{U}+\sum_{k=0}^{\mathbf{K}-1} \prod_{\ell=0}^{k-1} \mathbf{I}_{\ell}\left(1-\mathbf{I}_{k}\right)\left(k \tau+\mathbf{X}_{k}\right)+\prod_{\ell=0}^{\mathbf{K}-1} \mathbf{I}_{\ell} Z / v,
$$

with $\mathbf{K}=\lfloor(Z / v-\mathbf{U}) / \tau\rfloor$. Note that given $\mathbf{U}$, the random variables $\mathbf{I}_{k}$ are conditionally independent,

$$
\begin{gathered}
\mathrm{E}[\mathbf{D} \mid \mathbf{U}]=\mathbf{U}+\sum_{k=0}^{\mathbf{K}-1} q_{k}(\mathbf{U} v, v)(k \tau(1-p((\mathbf{U}+k \tau) v, v)) \\
+\mathrm{E}[\mathbf{X}]-x((\mathbf{U}+k \tau) v, v))+q_{\mathbf{K}}(v \mathbf{U}, v) Z / v,
\end{gathered}
$$

such that,

$$
\begin{aligned}
& \mathrm{E}[\mathbf{D}]=\int_{0}^{\tau}\left[u+q_{K(u)}(v u, v) Z / v+\sum_{k=0}^{K(u)-1} q_{k}(u v, v) \times\right. \\
& (k \tau \bar{p}((u+k \tau) v, v)+\mathrm{E}[\mathbf{X}]-x((u+k \tau) v, v))] \frac{d u}{\tau},
\end{aligned}
$$

with $\bar{p}(t, v)=1-p(t, v)$ and $K(u)=\lfloor(Z / v-u) / \tau\rfloor$ as before. Again approximating the uniform distribution of $\mathbf{U}$ by a discrete uniform distribution, we finally have,

$$
\begin{aligned}
& \mathrm{E}[\mathbf{D}] \approx \frac{\tau}{2}+\frac{1}{M} \sum_{m=0}^{M-1}\left[q_{K_{m}}\left(u_{m} v, v\right) \frac{Z}{v}+\sum_{k=0}^{K_{m}-1} q_{k}\left(u_{m} v, v\right) \times\right. \\
& \left.\left(k \tau \bar{p}\left(\left(u_{m}+k \tau\right) v, v\right)+\mathrm{E}[\mathbf{X}]-x\left(\left(u_{m}+k \tau\right) v, v\right)\right)\right],
\end{aligned}
$$


with $u_{m}=\frac{m \tau}{M}$ and $K_{m}=K\left(u_{m}\right)$. In view of the definition of $\mathbf{D}$, we can also calculate the mean time till discovery, conditioned on the message being discovered. We have,

$$
\mathrm{E}[\mathbf{D} \mid \text { discovered }]=\frac{1}{p_{d}}\left(\mathrm{E}[\mathbf{D}]-Z / v\left(1-p_{d}\right)\right),
$$

where $p_{d}$ is the discovery probability, see (4).

\section{Performance Evaluation}

We now illustrate our approach by some numerical examples. The values used correspond to the IEEE 802.11p/1609.4 setup and are summarized in Table I. For the locationdependent BER, we translate the packet error probabilities of [5] to bit errors, that is, we set

$$
b(z, v)=1-q(z)^{1 / L}
$$

with $L$ the packet size in bits and with $q(z)$ the location dependent probability that the packet is correctly received, the RSU being in position $600 \mathrm{~m}$ ( $z$ is expressed in meters),

$$
q(z)= \begin{cases}0.1 & \text { for } z \in[0,100) \cup[1100,1200) \\ \frac{210-\frac{2}{5}|600-z|}{100} & \text { for } z \in(100,200] \cup[1000,1100) \\ 0.999 & \text { for } z \in[200,1000) \\ 0 & \text { otherwise }\end{cases}
$$

Figs. 2, 3 and 4 show the utilization $\rho$, the inverse of the discovery probability $1-p_{d}$ and the mean time till discovery (in $\mathrm{ms}$ ), conditional on the service being discovered vs. the SAM period $\tau$ (in ms). For all figures, we depict the performance measures for various $N$ when location-dependent BER is and is not taken into account. In the latter case, we use the average BER in the interval $[0,1200)$. Clearly, when the SAM period $\tau$ increases, the utilization increases as less time is spent on discovery. As the channel utilization does not depend on the error model, see (2), the curves with locationdependent and constant BERs coincide. Moreover, if there are fewer contending vehicles, the utilization improves, as less time is spent on resolving contention. Fig. 3 shows that an increase of $\tau$ implies a decrease of the discovery probability. Indeed, for longer SAM periods there are fewer occasions to connect while the vehicle is in range. Moreover, the service is more easily discovered if there are fewer contending vehicles as the chance for service discovery in a single SAM period is higher. In addition, the effect of location-dependent BER is apparent, the model without constant BER underestimating $p_{d}$. Finally, the conditional mean time till discovery, first increases and then decreases again for increasing $\tau$ for the locationdependent BER model (for $N \in\{5,10,15\}$ ), while it only increase for the constant BER model. The increase is expected: if $\tau$ is longer, the time between discovery opportunities increases which leads to longer discovery times. However, if $\tau$ further increases, the probability that one cannot connect increases as well. As we only consider the conditional discovery time and the discovery time is typically shorter conditioned on discovery, we find that the conditional mean discovery time decreases for increasing $\tau$. Finally, for $N=20$ and the location-dependent BER model, the discovery probability in a single SAM period is considerably lower, such that the latter effect immediately dominates.
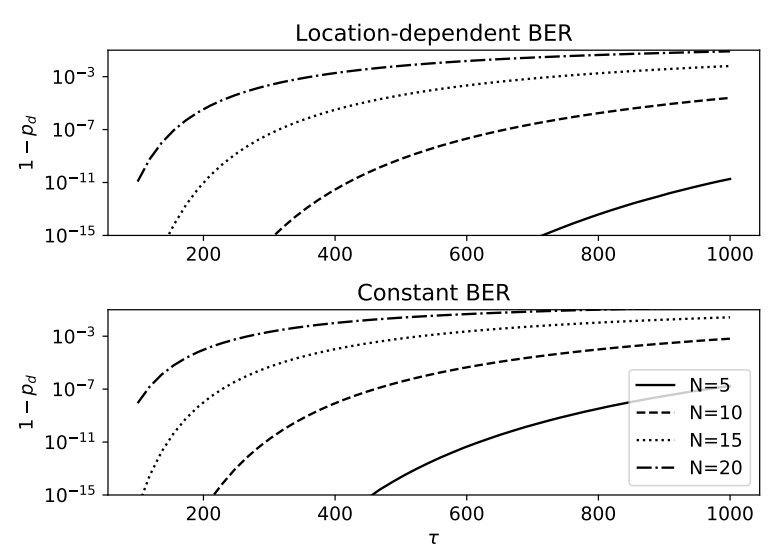

Fig. 3. Discovery probability $p_{d}$ vs. the SAM period $\tau$ for different numbers of vehicles $N$ as indicated, and for position dependent and constant BER.
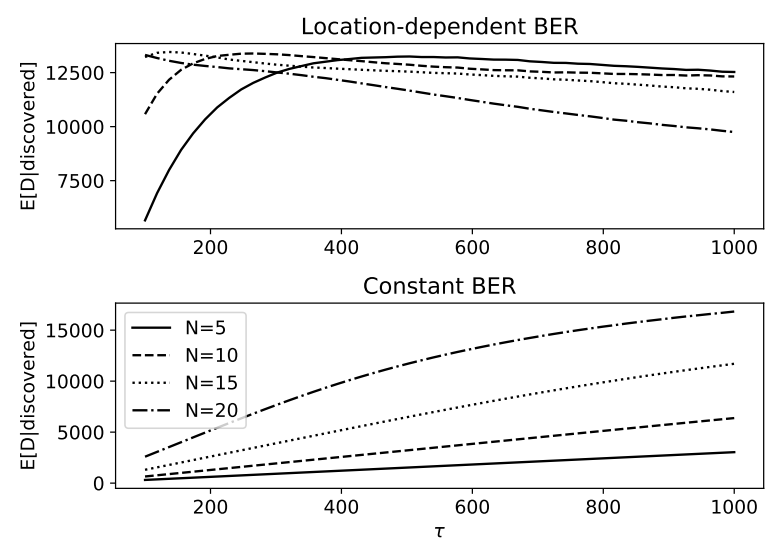

Fig. 4. Mean (conditional) discovery delay $E[\mathbf{D} \mid$ discovered $]$ vs. the SAM period $\tau$ for different numbers of vehicles $N$ as indicated, and for position dependent and constant BER.

\section{CONCLUSION}

We have proposed and analyzed a stochastic model for service discovery in a drive-through scenario. Accounting for location-dependent bit error probabilities, our model overcomes a fundamental limitation of prior work [4]. Our numerical results show that neglecting the effect of location on the transmission errors, may lead to substantial differences in discovery probabilities and mean discovery delays.

\section{REFERENCES}

[1] M. Xing et al. Maximum-utility scheduling for multimedia transmission in drive-thru Internet. IEEE Transactions on Vehicular Technology 65(4), pp.2649-2658, 2016.

[2] H. Zhou et al. Spatial coordinated medium sharing: optimal access control management in drive-thru internet. IEEE Transactions on Intelligent Transportation Systems 16(5), pp. 2673-2686, 2015.

[3] R.F. Atallah et al. Modeling and Performance Analysis of Medium Access Control Schemes for Drive-Thru Internet Access Provisioning Systems. IEEE Transactions on Intelligent Transportation Systems 16(6), pp. 3238-3248, 2015.

[4] C. Campolo et al. Service discovery and access in vehicle-to-roadside multi-channel VANETs. In Proc. of International Conference on Communication Workshop, IEEE, pp. 2477-2482, 2015.

[5] A. Böhm et al. Adaptive cooperative awareness messaging for enhanced overtaking assistance on rural roads. In Proc. of the 4th International Symposium on on Wireless Vehicular Communications. pp. 1-5, 2011. 